\title{
Estratégias de controle de Conyza spp. em pré-plantio da soja: Aplicações únicas ou
}

\section{sequenciais?}

\author{
Control strategies for Conyza spp. in soybean pre-planting: Single or sequential applications? \\ Estrategias de control para Conyza spp. en pre-siembra de soja: Aplicaciones únicas o
}

secuenciales?

Recebido: 09/06/2021 | Revisado: 17/06/2021 | Aceito: 22/06/2021 | Publicado: 07/07/2021

\author{
Paulo Vinicius da Silva \\ ORCID: https://orcid.org/0000-0003-4647-5602 \\ Universidade Federal da Grande Dourados, Brasil \\ E-mail: paulovsilva@ufgd.edu.br \\ Pedro Henrique de Souza Menezes da Costa \\ ORCID: https://orcid.org/0000-0003-3005-7748 \\ Universidade Federal da Grande Dourados, Brasil \\ E-mail: pedro.smc@hotmail.com \\ Matheus Vieira Barbosa de Oliveira \\ ORCID: https://orcid.org/0000-0001-8758-2389 \\ Universidade Federal da Grande Dourados, Brasil \\ E-mail: mateusvboliveira@gmail.com \\ Daniela Maria Barros \\ ORCID: https://orcid.org/0000-0003-0515-3316 \\ Universidade Federal da Grande Dourados, Brasil \\ E-mail: daniela.maria.barros11@gmail.com \\ Daniel Zamignan Molina \\ ORCID: https://orcid.org/0000-0003-0893-7925 \\ Universidade Federal da Grande Dourados, Brasil \\ E-mail: danielzamignanmolina82@gmail.com \\ Roque Dias de Carvalho \\ ORCID: https://orcid.org/0000-0001-5433-5373 \\ Universidade Federal da Grande Dourados, Brasil \\ E-mail: roquediasagro@gmail.com \\ Patrícia Andrea Monquero \\ ORCID: https://orcid.org/0000-0002-9123-1861 \\ Universidade Federal de São Carlos, Brasil \\ E-mail: pamonque@ufscar.br \\ Estela Maris Inácio \\ ORCID: https://orcid.org/0000-0003-3262-2819 \\ Engenheira Agrônoma Autônoma, Brasil \\ E-mail: estelainacio@hotmail.com
}

\begin{abstract}
Resumo
A Conyza spp. é uma espécie de difícil controle, principalmente em estágios fenológicos mais avançados demandando estratégias específicas de controle químico. O objetivo desse trabalho foi avaliar o controle de Conyza spp. através de uma aplicação do herbicida 2,4-D + glifosato, seguidas ou não, de aplicações sequenciais de herbicidas dessecantes de forma isolada e/ou associados a herbicidas pré-emergentes em pré-plantio da cultura da soja. O experimento foi realizado a campo em uma área com infestação natural de Conyza spp em delineamento experimental de blocos ao acaso com 4 repetições e 18 tratamentos. Foi efetuada uma aplicação de 2,4-D + glifosato $\left(975+1025 \mathrm{~g}\right.$ i.a. ha $\left.{ }^{-1}\right)$ e após 15 dias da aplicação inicial (DAI) foram realizadas as aplicações sequenciais: diquat (400 g i.a. ha ${ }^{-1}$ ); carfentrazone (30 g i.a. ha $\left.{ }^{-1}\right)$; glufosinato de amônio $\left(500 \mathrm{~g}^{\text {i.a. }} \mathrm{ha}^{-1}\right)$; saflufenacil $\left(49 \mathrm{~g}\right.$ i.a. ha $\left.{ }^{-1}\right)$ individualmente e associados com flumioxazina + imazetapir $\left(50+100\right.$ g i.a. ha $\left.{ }^{-1}\right)$; sulfentrazone + diuron $\left(245+490 \mathrm{~g}^{\text {i.a. }}\right.$ ha $\left.^{-1}\right)$ e diclosulam (29,4 g i.a. ha $\left.{ }^{-1}\right)$ e a testemunha sem aplicação de herbicida. Uma única aplicação 2,4-D + glifosato não foi efetiva no controle. As aplicações que contaram com carfentrazone foram ineficazes no controle. Diquat, saflufenacil e glufosinato de amônio, associados com herbicidas pré-emergentes (diclosulam, sulfentrazone + diuron e flumioxazim + imazethapyr), resultaram em controle superiores a 80\%. Carfentrazone não é uma alternativa para o controle de Conyza spp., diquat, amônio glufosinato e saflufenacil isolados e/ou associados com herbicidas préemergentes são viáveis no controle de Conyza spp.
\end{abstract}

Palavras-chave: Residual; Controle químico; Manejo integrado. 


\begin{abstract}
Conyza spp. it is a difficult species to control, especially in more advanced phenological stages requiring specific chemical control strategies. The objective of this work was to evaluate the control of Conyza spp. through an application of 2,4-D herbicide + glyphosate, followed or not, by sequential applications of desiccant herbicides in isolation and/or associated with pre-emergent herbicides in pre-planting of soybean crop. The experiment was carried out in the field in an area with natural infestation of Conyza spp. in a randomized block experimental design with 4 replications and 18 treatments. An application of 2,4-D + glyphosate $\left(975+1025 \mathrm{~g}\right.$ a.i. ha $\left.{ }^{-1}\right)$ was performed and 15 days after the initial application (DAI) sequential applications were performed: diquat (400 $\mathrm{g}$ a.i. ha ${ }^{-1}$ ); carfentrazone (30 g a.i. ha ${ }^{-1}$ ); glufosinate ammonium (500 g a.i. ha ${ }^{-1}$ ); saflufenacil (49 $\mathrm{g}$ a.i. ha $\mathrm{h}^{-1}$ ) individually and associated with flumioxazim + imazethapyr $\left(50+100 \mathrm{~g}\right.$ a.i. $\left.\mathrm{ha}^{-1}\right)$; sulfentrazone + diuron $\left(245+490 \mathrm{~g}\right.$ a.i. ha $\left.\mathrm{h}^{-1}\right)$ and diclosulam $(29.4$ $\mathrm{g}$ a.i. $\mathrm{ha}^{-1}$ ) and the control without herbicide application. A single 2,4-D + glyphosate application was not effective in the control. Applications that relied on carfentrazone were ineffective in controlling. Diquat, saflufenacil and ammonium glufosinate, associated with pre-emergent herbicides (diclosulam, sulfentrazone + diuron and flumioxazim + imazethapyr), resulted in a control greater than $80 \%$. Carfentrazone is not an alternative for the control of Conyza spp., diquat, glufosinate ammonium and saflufenacil isolated and/or associated with pre-emergent herbicides are viable in the control of Conyza spp.
\end{abstract}

Keywords: Residual; Chemical control; Integrated management.

\title{
Resumen
}

Conyza spp. es una especie de difícil control, especialmente en etapas fenológicas más avanzadas que requieren estrategias específicas de control químico. El objetivo del presente trabajo es evaluar el control de Conyza spp. con una aplicación de 2,4-D + glifosato, seguida o no, de aplicaciones secuenciales de herbicidas desecantes de forma aislada y / o asociados con herbicidas pre-emergentes en la pre-siembra del cultivo de soja. El experimento se realizó en campo en una área con infestación natural de Conyza spp en un diseño experimental de bloques completos al azar, con 4 repeticiones y 18 tratamientos. Se realizó aplicación de 2,4-D + glifosato $(975+1025$ g i.a. ha-1) y 15 días después de la aplicación inicial (DAI) se realizaron aplicaciones secuenciales: diquat (400 g i.a. ha $\left.{ }^{-1}\right)$; carfentrazona $\left(30 \mathrm{~g}\right.$ a.i. ha $\left.\mathrm{h}^{-1}\right)$; glufosinato de amonio $\left(500 \mathrm{~g}\right.$ a.i. $\left.\mathrm{ha}^{-1}\right)$; saflufenacil $\left(49 \mathrm{~g}\right.$ i.a. ha $\left.\mathrm{h}^{-1}\right)$ solos y asociados a flumioxazina + imazetapir $\left(50+100\right.$ g i.a. ha $\left.{ }^{-1}\right)$; sulfentrazona + diuron $\left(245+490\right.$ g i.a. ha $\left.{ }^{-1}\right)$ y diclosulam $\left(29,4\right.$ g i.a. ha $\left.^{-1}\right)$ y un testigo sin aplicación. Una sola aplicación de 2,4-D + glifosato no fue eficaz. Las aplicaciones que dependían de carfentrazona fueron ineficaces. Sin embargo, diquat, saflufenacil y glufosinato de amonio, asociados con herbicidas preemergentes (diclosulam, sulfentrazona + diuron y flumioxazim + imazethapyr), resultaron en un control superior al $80 \%$. Carfentrazona no es una alternativa para el control de Conyza spp., mientras diquat, glufosinato de amonio y saflufenacil aislados y / o asociados a herbicidas preemergentes son viables para control de Conyza spp.

Palabras clave: Residual; Control químico; Manejo integrado de malezas.

\section{Introdução}

As espécies de Conyza spp. (popularmente conhecidas como buva), pertencem à família Asteraceae, são originárias da América do Sul e apresentam ciclo anual, herbácea, com caule pouco ramificado e muito folhoso, folhas não dentadas eprolífera (Lorenzi, 2014). Conyza bonariensis tem capacidade de produzir em média 120 mil sementes (Kasparyet al., 2017; Piaseckiet al., 2019). Os propágulos possuem capítulos globosos, com pedúnculos florais e margeados por brácteas de coloração verde e os frutos do tipo aquênio, sendo uma característica vantajosa para a disseminação através do vento (Ulzurrunet al., 2020).

Essas características, associada aos fluxos de emergência de Conyza spp., devem ser considerados na tomada de decisão de medidas de manejo, em relação ao melhor controle e momento. Ulzurrunet al. (2020) estudando a reprodução de Conyza spp. (C. blakei, C. bonariensis, C. sumatrensis e C. lorentzii), constataram que C. bonariensis e C. sumatrensis obtiveram maior sucesso reprodutivo, exigindo um controle antecipado ainda em estágio fenológico inicial, pois nesse momento, medidas de controle, podem ser mais efetivas quando comparadas com estágio fenológicos mais avançados, que demandam estratégias de controle complementares.

Em áreas cultivadas com o sistema de sucessão soja/milho, o fluxo de emergência de Conyza sppse inicia a partir da colheita do milho safrinha até o momento da semeadura da soja, assim, o melhor momento de ação para o controle é no manejo outonal (época de pousio), entre as sucessões das culturas (Albrecht et al., 2019). No entanto, esse manejo em pré-plantio da 
cultura da soja, deve sempre estar associado a estratégias que visem a redução e/ou eliminação de novos fluxos germinativos das plantas daninhas na área agrícola, evitando assim o processo de re-infestação e mitigando a mato-competição entre Conyza spp. e a cultura da soja (Albrechtet al., 2020).

Em locais onde a espécie Conyza spp., apresentam estádios de desenvolvimento mais avançados ou em áreas em que essas plantas não foram controladas em uma única aplicação de herbicida, é necessário a realização de aplicações sequenciais. De acordo com estudos realizados pela Fundação MS na safra de soja 2018/2019, as aplicações sequenciais se destacaram no controle destas plantas daninhas, sendo que os herbicidas amônio glufosinato e saflufenacil posicionados após uma primeira aplicação de 2,4-D+glifosato, resultaram nas maiores porcentagens de controle (Grigolli, 2019).

Atualmente o complexo das buvas (C. sumatrensis; C. bonariensis e C. canadensis) infestam cerca de 11,8 milhões hectares no Brasil, com ocorrência em todos os estados brasileiros (Adegaset al., 2017). Em trabalho de Albrecht et al. (2019) observaram em áreas infestadas com 0,16 a 0,62 plantas $\mathrm{m}^{-2}$ de Conyza spp., uma redução de produtividade de soja 12,54 e 13,72\%, respectivamente. Além disso, Silva et al. (2014) verificaram que o período anterior a interferência (PAI) da cultura da soja com infestação da Conyza spp., foi de 24 dias após a emergência da cultura, período esse que pode ser superior em relação a outras espécies e condições edafoclimáticas dentre outros fatores.

Logo, o uso de herbicidas dessecantes associados aos pré-emergentes pode auxiliar na redução dos custos para produção, e promover um controle eficiente desses biotipos resistentes e/ou de plantas de Conyza spp. em estágios fenológicos avançados. Oliveira Neto et al. (2010), avaliando a eficácia de controle de Conyza bonariensis, observaram que os tratamentos com melhor porcentagem de controle geral aos 30 DAMI (dias após a aplicação do manejo de inverno) foram os que receberam os herbicidas residuais diclosulam e flumioxazin, com a associação dos herbicidas pós-emergentes glifosato+2,4-D, no manejo de inverno.

Dessa forma, no caso do controle químico, faz-se necessário a busca de alternativas eficientes para o controle de Conyza spp. O objetivo do presente trabalho foi avaliar o controle de Conyza spp. em pré-plantio da cultura da soja, através de uma primeira aplicação de 2,4-D + glifosato, seguidas ou não, de aplicações sequenciais de herbicidas dessecantes de forma isolada e/ou associados a herbicidas pré-emergentes na dessecação.

\section{Metodologia}

O experimento foi realizado em campo em área com infestação natural, contado com 22 plantas por $\mathrm{m}^{2}$ de Conyza spp., com plantas homogeneamente distribuídas em campo. No momento da aplicação, as plantas apresentavam altura superior a $20 \mathrm{~cm}$, segundo a escala numérica Biologische Bundesanstalt und Chemische Industrie (BBCH) proposta por Hess et al. (1997), classificadas como 50-59, o que equivale a emissão da inflorescência. Dessa forma no momento da aplicação as plantas estavam em estágio fenológico avançado.

O delineamento experimental utilizado foi ode blocos ao acaso, organizado em esquema fatorial $18 \times 9$, sendo dezoito tratamentos e nove períodos de avaliação, com quatro repetições para cada tratamento. As unidades experimentais foram constituídas de parcelas $3 \times 5$ metros, totalizando $15 \mathrm{~m}^{2}$ cada unidade experimental e $1440 \mathrm{~m}^{2}$ de área total do experimento. No momento da instalação do experimento foram coletadas amostras de solo para análise das características físico-químicas, os quais os dados podem ser observados na Tabela 1. 
Tabela 1. Resultado da análise química do solo.

\begin{tabular}{ccccccccccccc}
\hline $\mathbf{p H}\left(\mathbf{C a C l}_{2}\right)$ & $\mathbf{A l}$ & $\mathbf{H}+\mathbf{A l}$ & $\begin{array}{c}\mathbf{P} \\
(\mathbf{m e h l})\end{array}$ & $\mathbf{K}$ & $\mathbf{C a}$ & $\mathbf{M g}$ & $\mathbf{S B}$ & $\mathbf{C T C}$ & $\mathbf{V}$ & Areia & Silte & Argila \\
\hline 5,6 & 0 & 30,8 & 35,2 & 5,0 & 72,0 & 31,5 & 108,5 & 139,2 & 77,9 & 192 & 165 & 643 \\
\hline
\end{tabular}

Unidades: Al, $\mathrm{H}+\mathrm{Al}, \mathrm{Ca}, \mathrm{Mg}$, SB e CTC $\left(\mathrm{cmol}_{\mathrm{c}} \mathrm{dm}^{-3}\right) ; \mathrm{K}, \mathrm{P}(\mathrm{rmehl})\left(\mathrm{mg} \mathrm{dm}^{-3}\right) ; \mathrm{V}(\%)$. Fonte: Autores.

Os tratamentos de herbicidas que foram aplicados em pós-emergência das plantas de Conyza spp., são apresentados na Tabela 2.

Tabela 2. Tratamentos de herbicidas pós-emergentes aplicados para o controle de Conyza spp.

\begin{tabular}{|c|c|c|c|c|}
\hline Tratamentos & Inicial & Dose $^{1}$ & $10 \mathbf{D A A}^{2}$ & Dose \\
\hline 1 & 2,4-D + Glifosato & $975+1025$ & ------- & ------- \\
\hline 2 & 2,4-D + Glifosato & $975+1025$ & Diquat & 400 \\
\hline 3 & 2,4-D + Glifosato & $975+1025$ & Carfentrazone & 30 \\
\hline 4 & 2,4-D + Glifosato & $975+1025$ & Glufosinato de Amônio & 500 \\
\hline 5 & 2,4-D + Glifosato & $975+1025$ & Saflufenacil & 49 \\
\hline 6 & 2,4-D + Glifosato & $975+1025$ & Diquat + (fluimioxazina + imazetapir $)$ & $400+(150)$ \\
\hline 7 & 2,4-D + Glifosato & $975+1025$ & Carfentrazone + (fluimioxazina + imazetapir $)$ & $30+(150)$ \\
\hline 8 & 2,4-D + Glifosato & $975+1025$ & GS + (fluimioxazina + imazetapir) & $500+(150)$ \\
\hline 9 & 2,4-D + Glifosato & $975+1025$ & Saflufenacil + (flumioxazina + imazetapir) & $49+(150)$ \\
\hline 10 & 2,4-D + Glifosato & $975+1025$ & Diquat $+($ sulfentrazone + diurom $)$ & $400+(735)$ \\
\hline 11 & 2,4-D + Glifosato & $975+1025$ & Carfentrazone + (sulfentrazone + diurom $)$ & $30+(735)$ \\
\hline 12 & 2,4-D + Glifosato & $975+1025$ & $\mathrm{GS}+($ sulfentrazone + diurom $)$ & $500+(735)$ \\
\hline 13 & 2,4-D + Glifosato & $975+1025$ & Saflufenacil + (sulfentrazone + diurom $)$ & $49+(735)$ \\
\hline 14 & 2,4-D + Glifosato & $975+1025$ & Diquat + (diclosulan) & $400+(29,4)$ \\
\hline 15 & 2,4-D + Glifosato & $975+1025$ & Carfentrazone + (diclosulan $)$ & $30+(29,4)$ \\
\hline 16 & 2,4-D + Glifosato & $975+1025$ & GS + (diclosulan $)$ & $500+(29,4)$ \\
\hline 17 & 2,4-D + Glifosato & $975+1025$ & Saflufenacil + (diclosulan) & $49+(29,4)$ \\
\hline 18 & Testemunha & ------- & ------- & ------- \\
\hline
\end{tabular}

${ }^{1} \mathrm{~g}$ e.a ha ${ }^{-1}$ ou g i.a. ha ${ }^{-1}{ }^{2}$ Adição de óleo mineral. Fonte: Autores.

Os tratamentos de herbicidas foram aplicados com pulverizador costal pressurizado com $\mathrm{CO}_{2}$, com pressão de 2,0 bar, com barra de pulverização contendo seis pontas do tipo leque Teejet 110.015, espaçados com 0,5 m e com volume de aplicação de $150 \mathrm{~L} \mathrm{ha}^{-1}$. Na primeira aplicação a umidade relativa do ar era de $63,4 \%$, temperatura de $32,5^{\circ} \mathrm{C}$ e velocidade do vento de 2,6 $\mathrm{Km} \mathrm{h}^{-1}$ na segunda de $64,8 \%$, temperatura de $29,1^{\circ} \mathrm{C}$ e velocidade do vento de $0,8 \mathrm{Km} \mathrm{h}^{-1}$.

O controle percentual das plantas de Conyza spp. foi avaliado aos 15 dias após aplicação inicial (DAI), relacionada a aplicação de 2,4-D + glifosato e aos 7, 14, 21, 28, 35, 42, 49 e 56 dias após aplicação dos tratamentos (DAT) (relativas as aplicações sequenciais), seguindo a escala visual da ALAM (1974) na qual foi atribuído 0\% no caso da ausência de sintomas do herbicida e $100 \%$ para a morte das plantas.

Os resultados obtidos foram submetidos à análise de variância pelo teste $\mathrm{F}$, sendo as médias dos tratamentos comparadas pelo teste de Tukey, sendo utilizado o programa computacional AgroEstat (Barbosa \& Maldonado Júnior, 2021). 


\section{Resultados e Discussão}

Na Tabela 3 é possível visualizar a interação fatorial entre os períodos de avaliação e os tratamentos. Aos 15 DAI, onde ocorreu apenas à aplicação de 2,4-D + glifosato, não houve diferença estatística entre os tratamentos de herbicidas, sendo que em todos os tratamentos a porcentagem de controle foi inferior a 55\%, demonstrando homogeneidade do estágio fenológico das plantas no momento da aplicação dos tratamentos de herbicidas.

Tabela 3. Controle de Conyza spp. através de diferentes tratamentos de herbicidas.

\begin{tabular}{|c|c|c|c|c|c|c|c|c|c|}
\hline \multirow{3}{*}{ Tratamentos } & \multicolumn{9}{|c|}{ Períodos de Avaliação } \\
\hline & \multirow{2}{*}{$\begin{array}{c}15 \\
\text { DAI }\end{array}$} & 7 & 14 & 21 & 28 & 35 & 42 & 49 & 56 \\
\hline & & \multicolumn{8}{|c|}{ DAT } \\
\hline 1 & $52,5 \mathrm{aA}$ & $31,3 \mathrm{aAB}$ & $32,5 \mathrm{~dB}$ & $27,5 \mathrm{fB}$ & $36,3 \mathrm{aB}$ & $28,8 \mathrm{cB}$ & $26,3 \mathrm{aB}$ & $25 \mathrm{~dB}$ & $25 \mathrm{eB}$ \\
\hline 2 & $53,8 \mathrm{aB}$ & $88,8 \mathrm{abA}$ & $85 \mathrm{aA}$ & $81,3 \mathrm{abA}$ & 82,5 abcA & $93,8 \mathrm{aA}$ & $87 \mathrm{aA}$ & $86,3 \mathrm{aA}$ & $85 \mathrm{aA}$ \\
\hline 3 & $48,8 \mathrm{aA}$ & $36,3 \mathrm{dAB}$ & $32,5 \mathrm{bAB}$ & $26,3 \mathrm{fB}$ & $28,8 \mathrm{eB}$ & $25 \mathrm{cB}$ & $32,5 \mathrm{cAB}$ & $33,8 \mathrm{dAB}$ & $37,5 \mathrm{de} A B$ \\
\hline 4 & $51,3 \mathrm{aB}$ & $76,3 \mathrm{abcA}$ & $87,5 \mathrm{aA}$ & $83,8 \mathrm{abA}$ & $85 \mathrm{abA}$ & $87,5 \mathrm{aA}$ & $87,5 \mathrm{aA}$ & $85 \mathrm{abAB}$ & $85 \mathrm{aAB}$ \\
\hline 5 & $50 \mathrm{aB}$ & $88,8 \mathrm{abA}$ & $95 \mathrm{aA}$ & $87,5 \mathrm{aA}$ & $91,3 \mathrm{aA}$ & $95 \mathrm{aA}$ & $93,75 \mathrm{aA}$ & $91,3 \mathrm{aA}$ & $90 \mathrm{aA}$ \\
\hline 6 & $50 \mathrm{aC}$ & $80 \mathrm{abcAB}$ & $85 \mathrm{aA}$ & $73,8 \mathrm{abcB}$ & $76,3 \mathrm{abcAB}$ & $92,5 \mathrm{aA}$ & $90 \mathrm{aAB}$ & $82,5 \mathrm{abAB}$ & $82,5 \mathrm{abAB}$ \\
\hline 7 & $47,5 \mathrm{aA}$ & $45 \mathrm{dA}$ & $50 \mathrm{aA}$ & $45 \operatorname{def} A$ & $47,5 \mathrm{de} A$ & $42,5 \mathrm{cA}$ & $45 \mathrm{cA}$ & $45 \mathrm{cdA}$ & $50 \mathrm{cdA}$ \\
\hline 8 & $47,5 \mathrm{aC}$ & $70 \mathrm{bcB}$ & $83,8 \mathrm{aAB}$ & $82,5 \mathrm{abAB}$ & $82,5 \mathrm{abcAB}$ & $86,3 \mathrm{aAB}$ & $88,8 \mathrm{aA}$ & $87,5 \mathrm{aAB}$ & $87,5 \mathrm{aAB}$ \\
\hline 9 & $50 \mathrm{aB}$ & $91,3 \mathrm{aA}$ & $91,3 \mathrm{aA}$ & $86,3 \mathrm{aA}$ & $91,3 \mathrm{aA}$ & $95 \mathrm{aA}$ & $90 \mathrm{aA}$ & $90 \mathrm{aA}$ & $90 \mathrm{aA}$ \\
\hline 10 & $52,5 \mathrm{aB}$ & 87,5 abcA & $92,5 \mathrm{aA}$ & $83,8 \mathrm{abA}$ & $87,5 \mathrm{abA}$ & $98 \mathrm{aA}$ & $92,5 \mathrm{aA}$ & $89,5 \mathrm{aA}$ & $86,3 \mathrm{aA}$ \\
\hline 11 & $48,8 \mathrm{aA}$ & $46,3 \mathrm{dA}$ & $50 \mathrm{bA}$ & $41,3 \mathrm{efA}$ & $37,5 \mathrm{eA}$ & $36,3 \mathrm{cA}$ & $37,5 \mathrm{cA}$ & $37,5 \mathrm{dA}$ & $40 \mathrm{de} A$ \\
\hline 12 & $48,8 \mathrm{aC}$ & $67,5 \mathrm{cB}$ & $83,8 \mathrm{aAB}$ & $82,5 \mathrm{abAB}$ & $86,3 \mathrm{abA}$ & $93,8 \mathrm{aA}$ & $87,5 \mathrm{aA}$ & $86,3 \mathrm{aA}$ & $82,5 \mathrm{abA}$ \\
\hline 13 & $50 \mathrm{aB}$ & $90 \mathrm{abA}$ & $95 \mathrm{aA}$ & $91,3 \mathrm{aA}$ & $92,5 \mathrm{aA}$ & $95 \mathrm{aA}$ & $93,8 \mathrm{aA}$ & $93,8 \mathrm{aA}$ & $91,3 \mathrm{aA}$ \\
\hline 14 & $55 \mathrm{aC}$ & 78,8 abc $A B$ & $76,3 \mathrm{aAB}$ & $65 \mathrm{bcdBC}$ & $70 \mathrm{bcABC}$ & $80 \mathrm{abAB}$ & $75 \mathrm{abAB}$ & $81,3 \mathrm{abAB}$ & $87,5 \mathrm{aA}$ \\
\hline 15 & 51,3 aABC & $45 \mathrm{Dc}$ & $47,5 \mathrm{bBC}$ & 57,5 cdeABC & $62,5 \mathrm{cdABC}$ & $65 \mathrm{bAB}$ & $66,3 \mathrm{bA}$ & $65 \mathrm{bcAB}$ & $62,5 \mathrm{bcABC}$ \\
\hline 16 & $50 \mathrm{aB}$ & 81,3 abcA & $91,2 \mathrm{aA}$ & $82,5 \mathrm{abA}$ & $87,5 \mathrm{abA}$ & $94,8 \mathrm{aA}$ & $90 \mathrm{aA}$ & 88,8 aA & $87,5 \mathrm{aA}$ \\
\hline 17 & $53,8 \mathrm{aB}$ & $95 \mathrm{aA}$ & $95 \mathrm{aA}$ & $91,3 \mathrm{aA}$ & $90 \mathrm{abA}$ & $97,3 \mathrm{aA}$ & $93,8 \mathrm{aA}$ & $93,8 \mathrm{aA}$ & $93,8 \mathrm{aA}$ \\
\hline 18 & $0,00 \mathrm{bA}$ & $0,00 \mathrm{eA}$ & $0,00 \mathrm{cA}$ & $0 \mathrm{gA}$ & $0 \mathrm{fA}$ & $0 \mathrm{dA}$ & $0 \mathrm{dA}$ & $0 \mathrm{dA}$ & $0 \mathrm{fA}$ \\
\hline \multicolumn{3}{|c|}{$\mathbf{F}_{\text {tratamento }}=12,45^{* *}$} & \multicolumn{4}{|c|}{$\mathbf{F}_{\text {avaliação }}=81,98^{* *}$} & \multicolumn{3}{|c|}{$\mathbf{F}_{\text {tratamento } \times \text { avaliação }}=15,31^{* *}$} \\
\hline
\end{tabular}

${ }^{* *}$ Significativo ao nível de $1 \%$ de probabilidade pelo teste F; C.V. - Coeficiente de variação. Médias seguidas por letras iguais, minúsculas na coluna e maiúsculas na linha, não diferem entre si pelo teste de Tukey a 5\% de significância. Fonte: Autores.

Em relação a primeira avaliação, após a aplicação sequencial (7 DAT), podemos destacar os tratamentos $9(2,4-\mathrm{D}+$ glifosato com sequencial de saflufenacil + (flumioxazina + imazetapir) e 8 (2,4-D + glifosato com sequencial de GS + (flumioxazina + imazetapir), que apresentaramos maiores controles com 91,25 e 95\%, respectivamente.

Aos 56 DAT, o menor controle foi obtido no tratamento 1, o qual foi constituído por uma única aplicação de 2,4-D + glifosato com $25 \%$ de controle. Todos os tratamentos que constaram com a aplicação de carfentrazone, resultaram em controle ineficaz de Conyza spp., sendo que os tratamentos 3 (2,4-D + glifosato com sequencial de carfentrazone), 7 (2,4-D + glifosato com sequencial de carfentrazone + (flumioxazina + imazetapir) e $11(2,4-\mathrm{D}+$ glifosato com sequencial de carfentrazone + (sulfentraozne + diuron), não diferiram entre si, e apresentaram controles de 37,5, 50 e 40\%, respectivamente. Já o tratamento 15 (2,4-D + glifosato com sequencial de carfentrazone + (diclosulam), diferiu dos demais tratamentos com carfentrazone, no entanto, o controle de Conyza spp.também foi ineficaz com $62,5 \%$.

Os tratamentos $2,4,5,8,9,10,14,14,16$ e 17, aos 56 DAT não diferiram entre si na análise estatística, e apresentaram os melhores controles de Conyza spp. com porcentagens superiores a 85\%. Comparativamente, os tratamentos 6 
e 12, apresentaram porcentagens de controle de $82,5 \%$. Ou seja, todos os tratamentos com diquat, saflufenacil e glufosinato de amônio, associados com os herbicidas pré-emergentes, diclosulam, sulfentrazone + diuron e flumioxazim + imazethapyr.

O tratamento 2 (2,4-D + glifosato com sequencial de diquat), 5 (2,4-D + glifosato com sequencial de saflufenacil), 9 $(2,4-\mathrm{D}+$ glifosato com sequencial de saflufenacil + (flumioxazina + imazetapir), 10 (2,4-D + glifosato com sequencial de diquat + (flumioxazina + imazetapir), 13 (2,4-D + glifosato com sequencial de saflufenacil + (diuron + sulfentrazone), 16 (2,4D + glifosato com sequencial de glufosinato de amônio + diclosulan) e 17 (2,4-D + glifosato com sequencial de saflufenacil + diclosulam), não apresentam diferenças estatísticas ao longo das avaliações de controle posteriores a aplicação do 2,4-D + glifosato, sendo observadas porcentagens superiores a $80 \%$ nos diferentes períodos avaliados, sendo que todos os tratamentos que continham o saflufenacil na aplicação sequencial, estavam a inclusos nessa situação (5; $9 ; 13$ e 17).

O tratamento 1 (2,4-D + glifosato), foi o único que apresentou redução gradativa do 15 DAI até os 56 DAT. Já o tratamento 14 (2,4-D + glifosato com sequencial de diquat + diclosulam) apresentou evolução gradativa das porcentagens de controle ao longo dos períodos de avaliação.

Com os resultados obtidos, é possível observar que uma única aplicação da associação de 2,4-D + glifosato em plantas de Conyza spp. em estágio fenológico avançado, não é suficiente para se estabelecer as porcentagens de controle aceitáveis, (superiores a 80\%), uma vez que esse tratamento resulta em gradativa redução das porcentagens de controle. Esse comportamento está associado as rebrotas nas plantas de Conyza spp, indicando a necessidade de uma aplicação sequencial de herbicidas visando se estabelecer níveis adequados de controle.Segundo Oliveira Neto et al. (2013), todos os métodos de controle quando utilizados isoladamente tem se mostrado pouco eficientes, de tal maneira que sempre acabam selecionando novos problemas.

Oliveira Neto et al. (2010), mediante a aplicação de de 2,4-D + glifosato visando o controle de C. bonariensis, verificaram que o crescimento de $1 \mathrm{~cm}$ dessas plantas foi acompanhado da redução de 1,71\% nas porcentagens de controle aos 14 DAT, sendo que aos 35 DAT, associação de glyphosate + 2,4-D resultou em controle de 95\% para plantas com altura inferior a $6 \mathrm{~cm}$, e de $55 \%$ para as plantas com altura de $21 \mathrm{~cm}$. Esses resultados além de corroborar com os apresentados nessa pesquisa, também evidenciam que aplicações de glifosato + 2,4-D em plantas de Conyza spp. em estágios fenológicos mais avançados necessitam de uma segunda aplicação para resultar em níveis adequados de controle.

Nesse experimento o carfentrazone, não foi uma opção a ser utilizado em aplicações sequenciais no controle de Conyza spp., pois em todos os tratamentos que contaram com a sequencial desse herbicida isolado e/ou associado com os herbicidas pré-emergentes, as porcentagens de controle foram inferiores a $50 \%$.

Moreira et al. (2010), aplicando a associação de glifosato + carfentrazone $\left(1.440+30 \mathrm{~g}\right.$ i.a. ha $\left.{ }^{-1}\right)$ para o controle de Conyza spp., obtiveram controle de 31,6 e $10 \%$ para os estágios fenológicos de dez folhas e pré-florescimento aos 28 DAA. Isso acontece, pois a maior eficácia do carfentrazone está direcionada as espécies dos gêneros Commelina spp e Ipomoea spp. (Christoffoleti et al., 2016, Da Silva et al., 2019), portanto, independente do estádio das plantas de buva, este herbicida não será a melhor ferramenta no controle.

A associação de carfentrazone com os herbicidas pré-emergentes (sulfentrazone + diuron e imazethapyr + flumioxazim) resultou em efeito aditivo, pois não houve aumento da porcentagem de controle de Conyza spp., quando comparado com os resultados obtidos da aplicação do carfentrazone de forma isolada. Já a associação do carfentrazone com o herbicida diclosulam, resultou em efeito sinérgico, logo embora não haja registro para utilização do diclosulam em pósemergência, esse herbicida demostrou efeito nessa modalidade, pós-emergência, no presente experimento.

Rossi et al. (2018), verificaram que os tratamentos sequenciais (após a aplicação de 2,4-D + glifosato) com o herbicida diclosulam na composição, favoreceram a velocidade de controle, em especial quando associado ao paraquat, onde níveis de controle numericamente superiores a $90 \%$ foram observados aos 14 DAA, para aplicações iniciadas no período seco 
ou úmido, mostrando uma porcentagem de controle superior a aplicação do paraquat isolado na sequencial, o qual apresentou um controle de $85 \%$, ou seja, denotam um efeito sinérgico com adição de diclosulan na sequencial. Oliveira Neto et al. (2013), relatam que além de promover o controle em pós-emergência de Conyza spp. O diclosulam também tem o benefício do residual quando posicionado na dessecação, favorecendo o controle adequado dos novos fluxos de plantas daninhas.

Os herbicidas pós-emergentes diquat, amônio glufosinato e saflufenacil, representam uma excelente alternativa na aplicação sequencial de controle de Conyza spp, tanto isolado quanto associados com os pré-emergentes, representando uma alternativa ao herbicida paraquat que foi retirado do mercado brasileiro em 22 de setembro de 2020, e era frequentemente posicionado como dessecante na aplicação sequencial.

Todasos tratamentos que contaram com amônio glufosinato e saflufenacil na sequencial, isolados e/ou associados com herbicidas pré-emergentes resultaram em excelentes porcentagens de controle de Conyza spp. Outro efeito positivo na utilização desses herbicidas em aplicações sequenciais, pode ser associada ao rápido controle, ou seja, quando forma posicionado na sequencial, eles aceleram o controle de Conyza spp.

Esse efeito de controle mais rápido mediante ao posicionamento dos herbicidas saflufenacil e glufosinato de amônio, podem ser justificados pela translocação que esses herbicidas apresentam em aplicações na pós-emergência. No caso do saflufenacil, ele apresenta propriedades físico-químicas que permitem uma pequena mobilidade via floema (Francischini et al., 2020). O saflufenacil, apresenta pKa de 4,41 e log Kow de 2,6 que lhe conferem uma certa translocação via floema, que pode ser explicada pelo seu fraco caráter ácido (Dalazen et al., 2015, Rodrigues e Almeida, 2018). Essas características o colocam na região limítrofe da mobilidade no floema/ xilema e mobilidade apenas no xilema (Dalazen et al., 2015).

Takano et al. (2020), relatam que a translocação limitada de glufosinato é provavelmente relacionada às suas características físico-químicas, ao invés de sua rápida ação. Assim, a translocação ocorre principalmente através do apoplasto, indicando que o movimento do glufosinato é dependente da transpiração, pois esse herbicida tende a se acumular nas folhas velhas com maiores taxas de transpiração ao invés das folhas jovens e o meristema apical. A rápida ação do glufosinato não limita sua própria translocação. Em vez disso, baixas taxas de translocação resultam de a ausência de um transportador ativo eficiente.

Albrecht et al. (2020) aplicaram o tratamento glyphosate + 2,4-D $\left(1500+670 \mathrm{~g}\right.$ i.a. ha $\left.{ }^{-1}\right)$, com sequencial de amônio glufosinato (400 g i.a. ha ${ }^{-1}$ ) sobre plantas de Conyza ssp e observaram que em plantas com altura superior a $15 \mathrm{~cm}$ o controle foi de $83 \%$ aos 35 DAT. No mesmo período, todos os tratamentos que contaram em algum momento com aplicação de saflufenacil (35 g i.a. ha ${ }^{-1}$ ) apresentaram controle superior a 85\%. Ou seja, na estratégia de controle de Conyza ssp., os herbicidas amônio glufosianto e safluenacil apresentaram controle adequado para essa espécie.

Os herbicidas diquat, amônio glufosinato e saflufenacil, resultaram em efeito aditivo na associação com sulfentrazone + diuron, imazethapyr + flumioxazim e diclosulam, ou seja, não reduziram o efeito dos dessecantes utilizados de forma individual e ainda representam uma alternativa efetiva para o controle de Conyza spp., além de entregarem um residual para a emergência de fluxos germinativos de plantas daninhas. Outro benefício dessa associação entre herbicidas dessecantes e préemergentes é o ganho operacional, reduzindo uma entrada na aplicação na área e o tráfego de maquinários, otimizando o tempo de planejamento na lavoura (Oliveira Neto et al., 2013).

Em trabalho realizado por Brustolin et al. (2020) aplicando a associação glifosato + sulfentrazona + diuron (1080 + 262-525 $\mathrm{g} \mathrm{ha}^{-1}$ ) aos 42 dias após a aplicação dos tratamentos, obtiveram controle de $92 \%$ de Conyza spp, os autores ainda relatam que a aplicação isolada de sulfentrazona + diuron (262-525 $\mathrm{g} \mathrm{ha}^{-1}$ ) resultaram em controle insatisfatório, com porcentagens inferiores a 80\%. Zobiole et al. (2018), trabalhando estratégias de controle de Conyza spp., com aplicação (diclosulam + halauxifen-metil) + glifosato (para dose de diclosulan partir de 23 g i.a. ha ${ }^{-1}$ ) com sequencial de amônioglufosinato (366 g i.a. ha ${ }^{-1}$ ), paraquat (400 g i.a. ha ${ }^{-1}$ ) e saflufenacil (35 g i.a. ha ${ }^{-1}$ ), obtiveram os melhores níveis de 
controle, sendo superiores $89 \%$.

\section{Conclusão}

Em plantas de Conyza ssp. em florescimento, uma única aplicação da associação do herbicida 2,4 D + glifosato, não foi suficiente para resultar em controle, necessitando assim de uma aplicação sequencial. $O$ herbicida carfentrazone, não representou uma alternativa efetiva para o controle de Conyza ssp., em aplicações sequenciais, seja associado com herbicidas pré-emergentes ou isolado. Os herbicidas saflufenacil, amônio glufosinato e diquat, foram efetivos no controle de Conyza ssp., quando posicionados de forma sequencial, aplicados de forma isolada ou associados a herbicidas pré-emergentes, sendo que os herbicidas saflufenacil e amônio glufosinato aceleram o controle dessa espécie. O herbicida diclosulam apresentou efeito sinérgico quando associado ao carfentrazone na sequencial, demonstrando também efeito como um produto pós-emergente. Em relação as pesquisas futuras existem a necessidade de avaliar a eficiência no controle de fluxos de Conyza spp. através do posicionamento de herbicidas em diferentes momentos, e sua seletividade na cultura da soja.

\section{Agradecimentos}

A Fazenda Experimental de Ciências Agrárias da UFGD, pela disponibilidade da área e apoio na realização do experimento.

\section{Referências}

Adegas, F. S., Vargas, L., Gazziero, D. L. P., \& Karam, D. (2017) Impacto econômico da resistência de plantas daninhas a herbicidas no Brasil. CIRCULAR TÉCNICA - EMBRAPA, 132. <https://ainfo.cnptia.embrapa.br/digital/bitstream/item/162704/1/CT132-OL.pdf>.

Alam. (1974) Asociation Latino Americana de Malezas. Recomendaciones sobre unificación de los sistemas de evaluación em ensayos de control de malezas. Alam, 1, 35-38.

Albrecht, A. J. P., Pivetta, L. A., Albrecht, L. P., Silva, A. F. M., \& Pellizzaro, E. C. (2019) Atenção às dessecações e semeadura da soja. Revista campo \& Negócios, 18-19.

Albrecht, A. J. P., Albrecht, L. P., Silva, A. F. M., Ramos, R. A., Corrêa, N. B., Carvalho, M. G. De., Lorenzetti, J. B., \& Danilussi, M. T. Y. (2020) Control of Conyza spp. with sequential application of glufosinate in soybean pre-sowing. Ciência Rural, 50(9), e20190868.

Barbosa, J. C., Maldonado Júnior, W. AgroEstat versão 1.0 - Sistema de análises estatísticas de ensaios agronômicos. AgroEstat. 2021. $<$ https://www.agroestat.com.br/>. Acesso em: 25/01/2021

Brustolin, D. B., Muller, A. L., Kovaleski, S., Bernardi, D., Demartelaere, A. C. F., Feitosa, S. S., Medeiros, M. L. S., \& Silva, F. E. (2020) Associação de herbicidas como alternativa no controle e na avaliação do desempenho fisiológico da Conyza spp. antes da semeadura da Glycine max. Brazilian Journal of Development, 6(7), 51187-51198.

Christoffoleti, P. J., Nicolai, M., López-Ovejero, R. F., Borgato, E. A., Netto, A. G., \& Melo, M. S. C. (2016) Resistência de plantas daninhas a herbicidas: termos e definições importantes. In: Christoffoleti, P. J., Nicolai, M. (Org.). Aspectos de Resistência de Plantas Daninhas a Herbicidas. Piracicaba: Esalq, p. 11-31.

Da Silva, P. V., Barbosa, G. C., Ferrari, A., Tronquini, S. M., \& Monquero, P. A. (2019) Chemical control strategies of Commelina benghalensis in coffee crop. Coffee Science, 14, 231-239.

Dalazen, G., Kruse, N. D., Machado, S. L. D. O., \& Balbinot, A. (2015) Sinergismo na combinação de glifosato e saflufenacil para o controle de buva. Pesquisa Agropecuária Tropical, 45(2), 249-256.

Grigolli, J. F. J. (2019) Manejo e controle de plantas daninhas na cultura da soja. In: Lourenção, A. L. F., Gitti, D. C., Grigolli, J. F. J., Bezerra, A. R. G., Melotto, A. M. Tecnologia e Produção Soja Safra 2018/2019. Curitiba: Midiograf, p.130-146.

Francischini, A. C., Constantin, J., Matte, W. D., Oliveira Jr, R. S., Mendes, R. R., Machado, F. G., \& Silva, V. F. V. (2020) Controle químico associado à roçada de soqueiras do algodoeiro. Brazilian Journal of Development, 6(3), 15920-15934.

Hess, M., Barralis, G., Bleiholder, L., Buhr, L., Eggers, T. H., Hack, H., \& Stauss, R. (1997) Use of the extended BBCH scale - general for descriptions of the growth stages of mono and dicotyledonous weed species. Weed Research, 37(6), 433-441.

Kaspary, T. E., Lamego, F. P., Cutti, L., Aguiar, A. C. De M., Rigon, C. A. G., \& Basso, C. J. (2017) Growth, phenology, and seed viability between glyphosate-resistant and glyphosate-susceptible hary fleabane. Bragantia, 76(1), 92-101. 
Research, Society and Development, v. 10, n. 8, e10310816995, 2021

(CC BY 4.0) | ISSN 2525-3409 | DOI: http://dx.doi.org/10.33448/rsd-v10i8.16995

Lorenzi, H. (2014) Manual de identificação e controle de plantas daninhas: plantio direto e convencinal. Nova Odessa: Instituto Plantarum, 381 p.

Moreira, M. S., Melo, M. S. C., Carvalho, S. J. P., Nicolai, M., \& Crhistoffoleti, P. J. (2020) Herbicidas alternativos para o controle de biótipos de Conyza bonariensis e C. canadensis resistentes ao herbicida glyphosate. Planta Daninha, 28(1), p.167-175.

Oliveira Neto, A. M., Constantin, J., Oliveira Jr., R. S., Guerra, N., Dan, H. A., Alonso, D. G., Blainski, E., \& Santos, G. (2010) Estratégias de manejo de inverno e verão visando ao controle de Conyza bonariensis e Bidens pilosa. Planta Daninha, 28(spe), 1107-1116.

Oliveira Neto, A. M., Constantin, J., Oliveira Jr., R. S., Guerra, N., Dan, H. A., Vilela, L. M. S., Botelho, L. V. P., \& Ávila, L. A. (2013) Sistemas de dessecação de manejo com atividade residual no solo para áreas de pousio de inverno infestadas com buva. Comunicata Scientiae, 4(2), 120-128.

Piasecki, C., Mazon, A. S., Monge, A., Cavalcante, J. A., Agostinetto, D., \& Vargas, L. (2019) Glyphosate applied at the early reproductive stage impairs seed production of glyphosate-resistant hairy fleabane. Planta Daninha, 37.e019196815.

Rodrigues, B. N., \& Almeida, F. S. (2018) Guia de Herbicidas. (7a ed.), Produção Independente. 764p.

Rossi, C. V. S., Zobiole, L. H. S., Lucio, F. R., \& Neves, R. (2018) Comparação entre métodos químicos de controle de buva com início de dessecação em período seco ou úmido. Revista Brasileira de Herbicidas, 17(3), 1-9.

Silva, D. R. O., Vargas, L., Agostinetto, D., \& Mariani, F. (2014) Glyphosate-resistant hairy fleabane competition in RR® soybean. Bragantia, 73(4), 451457.

Takano, H., Beffa, R., Preston, C., Westra, P. \& Dayan, F. (2020) Glufosinate enhances the activity of protoporphyrinogen oxidase inhibitors. Weed Science, 68(4), 324-332.

Ulzurrun, P. D., Acedo, M. B., Garavano, M. E., Gianelli, V., \& Ispizua, V. N. (2020) Analysis of the agronomic interest characteristics for the management of Conyza blakei, Conyza bonariensis, Conyza sumatrensis, and Conyza lorentzii. Agrociencia Uruguay, 1(1), 1-15.

Zobiole, L. H. S., Krenchinski, F. H., Pereira, G. R., Rampazzo, P. E., Rubin, R. S., \& Lucio, F.R. (2018) Management programs to control Conyza spp. in pre-soybean sowing applications. Planta Daninha, 36.e018175883. 